\title{
DOUBLE COSINE-SINE SERIES AND NIKOL'SKII CLASSES IN UNIFORM METRIC
}

\begin{abstract}
In the this paper, we give neccessary and sufficient conditions for a function even with respect to the first argument but odd with respect to the second one to belong to the Nikol'skii classes defined by a mixed modulus of smoothness of a mixed derivative (both have arbitrary integer orders). These conditions involve the growth of partial sum of Fourier cosine-sine coefficients with power weights or the rate of decreasing to zero of these coefficients. A similar problem for generalized "small" Nikol'skii classes is also treated.
\end{abstract}

Key words: double cosine-sine series, mixed modulus of smoothness, Nikol'skii classes

2010 Mathematical Subject Classification: 42B05, 42B35, 42A32

1. Introduction. Let $\left\{a_{k}\right\}_{k=1}^{\infty}$ be a sequence of real numbers, such that $\sum_{k=1}^{\infty}\left|a_{k}\right|<\infty$. Then the functions

$$
f(x)=\sum_{k=1}^{\infty} a_{k} \cos k x
$$

and

$$
g(x)=\sum_{k=1}^{\infty} a_{k} \sin k x
$$

are continuous and $2 \pi$-periodic (i.e., $f, g \in C_{2 \pi}$ ) and (1) is the Fourier series of $f$, correspondingly, (2) is the Fourier series of $g$. Lorentz [13] established that the condition $\sum_{k=n}^{\infty}\left|a_{k}\right|=O\left(n^{-\alpha}\right), n \in \mathbb{N}$, for $0<\alpha<1$ 
implies $f \in \operatorname{Lip}(\alpha)$ or $g \in \operatorname{Lip}(\alpha)$. For $a_{k} \geqslant 0$, Boas [2] proved the following

Theorem 1. (i) Let $a_{k} \geqslant 0, k \in \mathbb{N}, 0<\alpha<1$ and $\left\{a_{k}\right\}_{k=1}^{\infty}$ be the sequence of Fourier sine or cosine coefficients of $\varphi$. Then $\varphi \in \operatorname{Lip}(\alpha)$ if and only if $\sum_{k=n}^{\infty} a_{k}=O\left(n^{-\alpha}\right), n \in \mathbb{N}$, or, equivalently, $\sum_{k=1}^{n} k a_{k}=O\left(n^{1-\alpha}\right)$, $n \in \mathbb{N}$.

(ii) If $a_{k} \geqslant 0, k \in \mathbb{N},\left\{a_{k}\right\}_{k=1}^{\infty}$ is the sequence of Fourier sine coefficients of $g$, then $g \in \operatorname{Lip}(1)$ if and only if $\sum_{k=1}^{\infty} k a_{k}<\infty$.

Similar result to (i) was earlier obtained by Rubinstein [19] (see also Theorem A in [26]).

Nemeth [15] established several generalizations of Theorem A and gave a sharp version of Theorem 3 from [2]. Dyachenko [6] studied trigonometric series with coeffcients of fractional order monotonicity, and obtained conditions for sums of such series to belong Lipschitz classes.

If $\omega$ is increasing and continuous on $[0 ; 2 \pi], \omega(0)=0$, then $\omega \in \Phi$. A function $\omega \in \Phi$ belongs to the Bary class $B$, if $\sum_{k=n}^{\infty} k^{-1} \omega\left(k^{-1}\right)=O\left(\omega\left(n^{-1}\right)\right)$, $n \in \mathbb{N}$; respectively, it belongs to the Bary-Stechkin class $B_{\alpha}, \alpha>0$, if $\sum_{k=1}^{n} k^{\alpha-1} \omega\left(k^{-1}\right)=O\left(n^{\alpha} \omega\left(n^{-1}\right)\right), n \in \mathbb{N}($ see [1]).

In the paper [3] by Butzer et al, several properties of fractional modulus of smoothness $\omega_{\beta}(f, \delta), \beta>0$, and its applications to the approximation theory were studied. Tikhonov [21], [22] proved a generalization of the Boas results in the case of fractional modulus of smoothness. In [23], the same author obtained the Boas type results for the Nikol'skii spaces $W^{\alpha} H_{\beta}^{\omega}$ of functions. Let us note, that the previous results of the Boas type connected with Nikol'skii classes belong to Chan [4] and Nemeth [16].

For multiple complex Fourier series one can note the papers by Móricz and Fülöp: [10] and [14]. Their results were generalized by the author of this paper in [26]. For the double cosine-sine series, Tevzadze [20] proved the following

Theorem 2. Let $m, n \in \mathbb{N}, a_{i k} \geqslant 0$ for all $i, k \in \mathbb{N}, \sum_{i, k=1}^{\infty} a_{i k}<\infty$, $h(x, y)=\sum_{i, k=1}^{\infty} a_{i k} \cos i x \sin k y$ and $\omega(t, \tau)$ be an increasing in each variable 
function on $[0,1]^{2}$, such that $\omega(0,0)=0$ and

$$
\begin{aligned}
& \int_{t}^{1} \int_{\tau}^{1} \omega(u, v) u^{-m-1} v^{-n-1} d u d v \leqslant C_{1} \omega(t, \tau) t^{-m} \tau^{-n}, \quad t, \tau \in(0,1] \\
& \int_{0}^{t} \int_{\tau}^{1} \omega(u, v) u^{-1} v^{-n-1} d u d v \leqslant C_{2} \omega(t, \tau) \tau^{-n}, \quad t, \tau \in(0,1], \\
& \int_{t}^{1} \int_{0}^{\tau} \omega(u, v) u^{-m-1} v^{-1} d u d v \leqslant C_{3} \omega(t, \tau) t^{-m}, \quad t, \tau \in(0,1], \\
& \int_{0}^{t} \int_{0}^{\tau} \omega(u, v) u^{-1} v^{-1} d u d v \leqslant C_{4} \omega(t, \tau), \quad t, \tau \in(0,1] .
\end{aligned}
$$

Then $h \in H^{m, n}(\omega)$ (see the next section) if and only if

$$
\sum_{i=[p / 2]}^{p} \sum_{k=[q / 2]}^{q} a_{i k} \leqslant C \omega(1 / p, 1 / q), \quad p, q \in \mathbb{N} \cap[2,+\infty)
$$

Similar results were obtained in [20] for double sine and cosine series. Fülöp (see [8] and [9]) gave the necessary and sufficient conditions for sums of sine, cosine, and mixed double series to belong the space $\Lambda_{*}(2)=H^{2,2}\left(\omega_{1,1}\right)$ (see the next section), where $\omega_{\alpha, \beta}(u, v)=u^{\alpha} v^{\beta}$, $0<\alpha, \beta \leqslant 1$. Donskikh [5] proved some multidimesional analogues of Fülöp results. Results from [8] and [9] were generalized by $\mathrm{Yu}$ [28] for classes $H H^{\omega}=H^{1,1}(\omega)$ (see the next section), where $\omega$ satisfies the conditions similar to (3)-(6) in the case $m=n=1$. Han, Li, and $\mathrm{Yu}$ [11] considered mixed modulus of smoothness of natural orders and obtained

Theorem 3. Let $\omega(u, v)$ be a conitinuous on $[0,2 \pi]^{2}$, increasing and subadditive in each variable function, such that $\omega(0,0)=0, a_{j k} \geqslant 0$ for all $j, k \in \mathbb{N}, \sum_{j=1}^{\infty} \sum_{k=1}^{\infty} a_{j k}<\infty, r, s \in \mathbb{N}$.

(i) If the following relations

$$
\sum_{j=1}^{m} \sum_{k=1}^{n} j^{r} k^{s} a_{j k}=O\left(m^{r} n^{s} \omega(1 / m, 1 / n)\right), \quad m, n \in \mathbb{N}
$$




$$
\begin{aligned}
& \sum_{j=1}^{m} \sum_{k=n+1}^{\infty} j^{r} a_{j k}=O\left(m^{r} \omega(1 / m, 1 / n)\right), \quad m, n \in \mathbb{N} ; \\
& \sum_{j=m+1}^{\infty} \sum_{k=1}^{n} k^{s} a_{j k}=O\left(n^{s} \omega(1 / m, 1 / n)\right), \quad m, n \in \mathbb{N} \\
& \sum_{j=m+1}^{\infty} \sum_{k=n+1}^{\infty} a_{j k}=O(\omega(1 / m, 1 / n)), \quad m, n \in \mathbb{N} ;
\end{aligned}
$$

are valid, then $g(x, y)=\sum_{k=1}^{\infty} \sum_{k=1}^{\infty} a_{j k} \sin j x \sin k y$ belongs to $H^{m, n}(\omega)$.

(ii) If $g \in H^{m, n}(\omega)$, then

$$
\sum_{j=1}^{m} \sum_{k=1}^{n} j^{r^{*}} k^{s^{*}} a_{j k}=O\left(m^{r^{*}} n^{s^{*}} \omega(1 / m, 1 / n)\right),
$$

where $m, n \in \mathbb{N}, r^{*}=r+1$ for even $r$ and $r^{*}=r$ for odd $r$.

Using several conditions on $\omega$, the authors of [11] obtained some criteria for $g \in H^{m, n}(\omega)$ in terms of Fourier coefficients of $g$, but the confusion in formula numeration in [11, Theorem $\mathrm{C}]$ makes understanding of statements hard. The conditions (7)-(10) are not independent (see Lemmas 2 and 4 below). Using these facts, results of Theorem 3 were rewritten in a new form and extended to the Nikol'skii classes in [27].

In the present study, we extend Theorem 3 and its counterpart from [27] to the case of differentiable even with respect to the first argument and odd with respect to the second one functions, using the mixed modulus of smoothness and derivatives of arbitrary natural orders (Theorem 4). In the case of weak monotone Fourier coefficients with a given rate of decreasing, we give the sharp conditions for $h$ from Theorem 2 to belong to the Nikol'skii classes $W^{r, s} H^{m, n}(\omega)$ (Theorem 5). Finally, we obtain an $o$-analogue of Theorem 4 (Theorem 6 ). The results are dependent on the evenness of $m+r$ and $n+s$.

2. Definitions. Let $r, s \in \mathbf{Z}_{+}=\{0,1, \ldots\},\left\{a_{j k}\right\}_{j, k \in \mathbb{N}} \subset \mathbb{R}$ and

$$
\sum_{j=1}^{\infty} \sum_{k=1}^{\infty} j^{r} k^{s}\left|a_{j k}\right|<\infty \text {. }
$$

It follows From (11) that the series

$$
\sum_{j=1}^{\infty} \sum_{k=1}^{\infty} a_{j k} \cos j x \sin k y
$$


and

$$
\sum_{j=1}^{\infty} \sum_{k=1}^{\infty} j^{r} k^{s} a_{j k} \cos (j x+\pi r / 2) \sin (k y+\pi s / 2)
$$

converge absolutely and uniformly to functions $h(x, y)$ and $\psi(x, y)$, respectively. By the classical theorem on differentiabilty of function series, we have

$$
h^{(r, s)}(x, y):=\frac{\partial^{r+s} h(x, y)}{\partial x^{r} \partial y^{s}}=\psi(x, y)
$$

everywhere. Let

$$
\dot{\triangle}_{t, \tau}^{m, n} f(x, y)=\sum_{j=0}^{m} \sum_{k=0}^{n}(-1)^{j+k}\left(\begin{array}{c}
m \\
j
\end{array}\right)\left(\begin{array}{l}
n \\
k
\end{array}\right) f(x+(m-2 j) t / 2, y+(n-2 k) \tau / 2)
$$

be the mixed difference of orders $m, n$ with steps $t, \tau$. For $\dot{\triangle}_{t, \tau}^{m, n} h^{(r, s)}(x, y)$ see Lemma 5. Let us consider the class $\Phi^{(2)}$ of positive on $[0,2 \pi]^{2} \backslash\{(0,0)\}$ functions $\omega$, for which $\omega(0,0)=0, \omega\left(x_{1}, y_{1}\right) \leqslant \omega\left(x_{2}, y_{1}\right), \omega\left(x_{1}, y_{1}\right) \leqslant \omega\left(x_{1}, y_{2}\right)$ if $x_{2} \geqslant x_{1}, y_{2} \geqslant y_{1}, x_{i}, y_{i} \in[0,2 \pi], i=1,2$.

If $\omega \in \Phi^{(2)}$ is such that

$$
\sum_{i=m}^{\infty} \sum_{j=n}^{\infty}(i j)^{-1} \omega\left(\frac{2 \pi}{i}, \frac{2 \pi}{j}\right)=O\left(\omega\left(\frac{2 \pi}{m}, \frac{2 \pi}{n}\right)\right), \quad m, n \in \mathbb{N},
$$

then $\omega$ belongs to the class $B B$.

If $m, n>0$ and for $\omega \in \Phi^{(2)}$ the inequality

$$
\sum_{i=1}^{j} \sum_{k=1}^{l} i^{m-1} k^{n-1} \omega\left(\frac{2 \pi}{i}, \frac{2 \pi}{k}\right)=O\left(j^{m} l^{n} \omega\left(\frac{2 \pi}{j}, \frac{2 \pi}{l}\right)\right), \quad j, l \in \mathbb{N},
$$

is valid, then $\omega$ belongs to the class $B_{m} B_{n}$. One-dimensional analogues of these classes were introduced by Bary and Stechkin [1]; for the twodimensional case see, for example, [28]. For $m, n \in \mathbb{N}$ and $\omega \in \Phi^{(2)}$, we will write $f \in H^{m, n}(\omega)$, if for all $\delta_{1}, \delta_{2} \in[0,2 \pi]$ the inequality $\omega_{m n}\left(f, \delta_{1}, \delta_{2}\right):=\sup \left\{\left|\dot{\triangle}_{t, \tau}^{m, n} f(x, y)\right|: 0 \leqslant t \leqslant \delta_{1}, 0 \leqslant \tau \leqslant \delta_{2}\right\} \leqslant C \omega\left(\delta_{1}, \delta_{2}\right)$ holds and $f \in W^{r, s} H^{m, n}(\omega), r, s \in \mathbb{Z}_{+}$, if $f^{(r, s)}$ exists everywhere and belongs to $H^{m, n}(\omega)$. Learn more about these classes in $L^{p}$ setting, e.g., in [18]. We will also consider

$$
h^{m, n}(\omega)=\left\{f \in H^{m, n}(\omega): \omega_{m n}\left(f, \delta_{1}, \delta_{2}\right)=o\left(\omega\left(\delta_{1}, \delta_{2}\right)\right)\right\},
$$


where $\delta_{1}, \delta_{2} \rightarrow 0+$, and $W^{r, s} h^{m, n}(\omega)$ are defined similarly to $W^{r, s} H^{m, n}(\omega)$. In the case $r=s=0, m=n=1$ and $\omega_{\alpha, \beta}(u, v)=u^{\alpha} v^{\beta}, 0<\alpha, \beta \leqslant 1$, we denote $W^{r, s} H^{m, n}\left(\omega_{\alpha, \beta}\right)$ by $\operatorname{Lip}(\alpha, \beta)$.

We shall write $\omega \in \Delta_{2}$, if $\omega(2 t, \tau) \leqslant C_{1} \omega(t, \tau)$ for all $2 t, \tau \in[0,2 \pi]$ and $\omega(t, 2 \tau) \leqslant C_{1} \omega(t, \tau)$ for all $t, 2 \tau \in[0,2 \pi]$.

3. Auxiliary propositions. Lemmas $1-4$ are proved in [26].

Lemma 1. Let $m, n>0, \omega \in \Phi^{(2)}$.

(i) If $\omega \in B_{m} B_{n}$, then $\omega \in \Delta_{2}$.

(ii) If $\omega \in B B \cap \Delta_{2}$, then $\omega(\cdot, t) \in B$ for any fixed $t \in[0,2 \pi]$.

Lemma 2. Let $\left\{a_{j k}\right\}_{j, k \in \mathbb{N}} \subset \mathbb{R}_{+}=[0,+\infty), \omega \in \Phi^{(2)}, m, n>0$.

(i) If $\omega \in B_{m} B_{n}$, then the condition

$$
\sum_{j=M}^{\infty} \sum_{k=N}^{\infty} a_{j k}=O\left(\omega\left(\frac{2 \pi}{M}, \frac{2 \pi}{N}\right)\right), \quad M, N \in \mathbb{N}
$$

implies

$$
\sum_{j=1}^{M} \sum_{k=1}^{N} j^{m} k^{n} a_{j k}=O\left(M^{m} N^{n} \omega\left(\frac{2 \pi}{M}, \frac{2 \pi}{N}\right)\right), \quad M, N \in \mathbb{N} .
$$

(ii) If $\omega \in B B \cap \Delta_{2}$, then (14) follows from (15).

Lemma 3. Let $\left\{a_{j k}\right\}_{j, k \in \mathbb{N}} \subset \mathbb{R}_{+}, \omega \in \Phi^{(2)}, m, n>0$.

(i) If $\omega \in B_{m} B_{n},\left\{a_{j k}\right\}_{j, k \in \mathbb{N}}$ satisfies (14) and

$$
\sum_{j=M}^{\infty} \sum_{k=N}^{\infty} a_{j k}=o\left(\omega\left(\frac{2 \pi}{M}, \frac{2 \pi}{N}\right)\right), \quad M, N \rightarrow \infty,
$$

then

$$
\sum_{j=1}^{M} \sum_{k=1}^{N} j^{m} k^{n} a_{j k}=o\left(M^{m} N^{n} \omega\left(\frac{2 \pi}{M}, \frac{2 \pi}{N}\right)\right), \quad M, N \rightarrow \infty .
$$

(ii) If $\omega \in B B \cap \Delta_{2},\left\{a_{j k}\right\}_{j, k \in \mathbb{N}}$ satisfies (17), then (16) is valid.

Lemma 4. (i) Let $\left\{a_{j k}\right\}_{j, k \in \mathbb{N}} \subset \mathbb{R}_{+}, \omega \in \Phi^{(2)}, \omega(\cdot, t) \in B$ for all $t \in[0,2 \pi], m, n>0$ and the relation (15) is valid. Then

$$
\sum_{j=M}^{\infty} \sum_{k=1}^{N} k^{n} a_{j k}=O\left(N^{n} \omega\left(\frac{2 \pi}{M}, \frac{2 \pi}{N}\right)\right), \quad M, N \in \mathbb{N} .
$$


(ii) If, instead of (15) in (i) we have (17), then

$$
\sum_{j=M}^{\infty} \sum_{k=1}^{N} k^{n} a_{j k}=o\left(N^{n} \omega\left(\frac{2 \pi}{M}, \frac{2 \pi}{N}\right)\right), \quad M, N \rightarrow \infty
$$

Lemma 5. If $m, k \in \mathbb{N}, \dot{\Delta}_{t}^{m} f(x)=\sum_{j=0}^{m}(-1)^{j}\left(\begin{array}{c}m \\ j\end{array}\right) f(x+(m-2 j) t / 2)$, then we have

$$
\begin{aligned}
& \dot{\Delta}_{t}^{m} \cos k x=(2 \sin k t / 2)^{m} \cos \left(k x+\frac{m \pi}{2}\right), \\
& \dot{\Delta}_{t}^{m} \sin k x=(2 \sin k t / 2)^{m} \sin \left(k x+\frac{m \pi}{2}\right) .
\end{aligned}
$$

In particular, for $h$ defined as the sum of (12) and $\psi$ defined as the sum of (13), respectively, under condition (11), we obtain

$$
\begin{aligned}
& \dot{\Delta}_{t, \tau}^{m, n} h(x, y)=\sum_{j=1}^{\infty} \sum_{k=1}^{\infty} a_{j k} \cos \left(j x+\frac{m \pi}{2}\right) \sin \left(k y+\frac{n \pi}{2}\right) \times \\
& \quad \times(2 \sin j t / 2)^{m}(2 \sin k \tau / 2)^{n} . \\
& \dot{\Delta}_{t, \tau}^{m, n} \psi(x, y)=\sum_{j=1}^{\infty} \sum_{k=1}^{\infty} j^{r} k^{s} a_{j k} \times \\
& \times \cos \left(j x+\frac{(m+r) \pi}{2}\right) \sin \left(k y+\frac{(n+s) \pi}{2}\right)(2 \sin j t / 2)^{m}(2 \sin k \tau / 2)^{n} .
\end{aligned}
$$

Proof. Let $\dot{\Delta}_{t}^{m} f(x)$ be as above. It is known that $\dot{\Delta}_{t}^{m} f(x)=\dot{\Delta}_{t}^{1}\left(\dot{\Delta}_{t}^{m-1} f(x)\right)$ and that $\dot{\Delta}_{t}^{1} e^{i k x}=(2 i \sin k t / 2) e^{i k x}$; therefore,

$$
\dot{\Delta}_{t}^{m} \cos k x=\operatorname{Re}\left(\dot{\Delta}_{t}^{m} e^{i k x}\right)=\operatorname{Re}\left[(2 i \sin k t / 2)^{m} e^{i k x}\right] .
$$

The last expression equals to $(-1)^{m / 2} \cos k x(2 \sin k t / 2)^{m}$ for even $m$ and to $(-1)^{(m+1) / 2} \sin k x(2 \sin k t / 2)^{m}$ for odd $m$. Thus, $\dot{\Delta}_{t}^{m} \cos k x=(2 \sin k t / 2)^{m} \times$ $\times \cos (k x+m \pi / 2)$. The second formula is proved in a similar manner. Since $\dot{\Delta}_{t, \tau}^{m, n} h(x, y)$ is the composition of $\dot{\Delta}_{t}^{m}$ with respect to $x$ and $\dot{\Delta}_{\tau}^{n} f$ with respect to $y$, we obtain (18). But differentiation and the $m$-th difference commute, also the equalities $\cos ^{(r)}(x)=\cos (x+r \pi / 2), \sin ^{(r)}(x)=\sin (x+$ $r \pi / 2$ ) hold, hence (19) is valid. 
Lemma 6. Let $r, s \in \mathbb{Z}_{+}$, $f$ be $2 \pi$-periodic in each variable continuous function. If $f^{(r, s)}$ exists everywhere and is continuous, then for any $m, n \in \mathbb{N}$ one has

$$
\omega_{m+r, n+s}\left(f, \delta_{1}, \delta_{2}\right) \leqslant \delta_{1}^{r} \delta_{2}^{s} \omega_{m n}\left(f^{(r, s)}, \delta_{1}, \delta_{2}\right), \quad \delta_{1}, \delta_{2} \in[0,2 \pi] .
$$

Lemma 6 may be proved in the same way as similar one-dimensional result (see $[25$, Ch.3, §3.3, (1)]) using representation of higher order difference by means of derivative (see $[25, \mathrm{Ch} .3, \S 3.3,(4)]$ ) and the equality of type $\dot{\Delta}_{h}^{m+r}=\dot{\Delta}_{h}^{r}\left(\dot{\Delta}_{h}^{m}\right)$ with respect to both variables. For this lemma in the case of mixed $L^{p}$ moduli of smoothness see [17] or [18].

\section{Main results}

Theorem 4. (i) Assume that $r, s \in \mathbb{Z}_{+},\left\{a_{j k}\right\}_{j, k \in \mathbb{N}}$ satisfies the condition (11) and $h(x, y)$ is the sum of (12). If $m, n \in \mathbb{N}, \omega \in B B \cap \Delta_{2}$ and the condition

$$
\sum_{j=1}^{M} \sum_{k=1}^{N} j^{m+r} k^{n+s}\left|a_{j k}\right|=O\left(M^{m} N^{n} \omega\left(\frac{2 \pi}{M}, \frac{2 \pi}{N}\right)\right), \quad M, N \in \mathbb{N},
$$

holds, then $h \in W^{r, s} H^{m, n}(\omega)$.

(ii) Let $\left\{a_{j k}\right\}_{j, k \in \mathbb{N}} \subset \mathbb{R}_{+}=[0, \infty)$ satisfy the condition (11), $h(x, y)$ be the sum of (12), $m, n, r, s$ be as in the part (i). If $\omega \in B B \cap \Delta_{2}$ and $m+r$ is even, $n+s$ is odd, then from $h \in W^{r, s} H^{m, n}(\omega)$ it follows that the condition (20) is valid. If $m+r$ is odd or $n+s$ is even and $\omega \in B_{m} B_{n} \cap B B$, then $h \in W^{r, s} H^{m, n}(\omega)$ also implies (20).

Proof. (i) Let the condition (20) be valid. By (19) from Lemma 5, we have for $t, \tau>0$ an upper estimate for $\left|\dot{\Delta}_{t, \tau}^{m, n} h^{(r, s)}(x, y)\right|$ of the type

$$
\begin{aligned}
2^{m+n} & \left(\sum_{j=1}^{M} \sum_{k=1}^{N}+\sum_{j=M+1}^{\infty} \sum_{k=1}^{N}+\sum_{j=1}^{M} \sum_{k=N+1}^{\infty}+\sum_{j>M} \sum_{k>N}\right) j^{r} k^{s}\left|a_{j k}\right| \times \\
& \times\left|\sin \frac{j t}{2}\right|^{m}\left|\sin \frac{k \tau}{2}\right|^{n}=: I_{M N}^{(1)}+I_{M N}^{(2)}+I_{M N}^{(3)}+I_{M N}^{(4)},
\end{aligned}
$$

where $M=[2 \pi / t], N=[2 \pi / \tau]$. By virtue of the inequality $|\sin x| \leqslant|x|$, $x \in \mathbb{R}$, and (20), we find that for $t, \tau \in(0,2 \pi]$

$$
I_{M N}^{(1)} \leqslant t^{m} \tau^{n} \sum_{j=1}^{M} \sum_{k=1}^{N} j^{m+r} k^{n+s}\left|a_{j k}\right|=O(\omega(t, \tau)) .
$$


Using Lemma 5, inequalities $|\sin x| \leqslant 1$ and $|\sin x| \leqslant|x|, x \in \mathbb{R}$, we have

$$
I_{M N}^{(2)} \leqslant 2^{m} \tau^{n} \sum_{j=M+1}^{\infty} \sum_{k=1}^{N} j^{r} k^{n+s}\left|a_{j k}\right| .
$$

One may rewrite (20) in the form

$$
\sum_{j=1}^{M} \sum_{k=1}^{N} j^{m} k^{n}\left(j^{r} k^{s}\left|a_{j k}\right|\right)=O\left(M^{m} N^{n} \omega\left(\frac{2 \pi}{M}, \frac{2 \pi}{N}\right)\right), \quad M, N \in \mathbb{N},
$$

and by Lemma 1(ii) and Lemma 4(i) we obtain

$$
\sum_{j=M+1}^{\infty} \sum_{k=1}^{N} k^{n}\left(j^{r} k^{s}\left|a_{j k}\right|\right)=O\left(N^{n} \omega\left(\frac{2 \pi}{M}, \frac{2 \pi}{N}\right)\right), \quad M, N \in \mathbb{N} .
$$

From (22) and (24) we deduce that $I_{M N}^{(2)}=O(\omega(t, \tau)), t, \tau \in[0,2 \pi]$. Similarly, we estimate $I_{M N}^{(3)}$. Finally, by the condition $\omega \in B B \cap \Delta_{2}$ and part (ii) of Lemma 2 (we again write (20) in the form (23))

$$
I_{M N}^{(4)} \leqslant \sum_{j=M+1}^{\infty} \sum_{k=N+1}^{\infty} 2^{m+n} j^{r} k^{s}\left|a_{j k}\right|=O(\omega(t, \tau)), t, \tau \in[0,2 \pi] .
$$

Combining the obtained estimates, we see that

$$
\left|\Delta_{t, \tau}^{m, n} h^{(r, s)}(x, y)\right|=O(\omega(t, \tau)), \quad t, \tau \in[0,2 \pi],
$$

whence the statement $h \in W^{r, s} H^{m, n}(\omega)$ follows.

(ii) In Steps I-IV, we assume that $r=s=0$ and set $t=M^{-1}, \tau=N^{-1}$ for $M, N \in \mathbb{N}$.

Step I. Let $m$ be even, $n$ be odd. Then, by Lemma 5 , we have

$$
C_{1} \omega(t, \tau) \geqslant\left|\dot{\Delta}_{t, \tau}^{m, n} h(0, v)\right|=\left|\sum_{j=1}^{\infty} \sum_{k=1}^{\infty} a_{j k}(2 \sin j t / 2)^{m}(2 \sin k \tau / 2)^{n} \cos k v\right| .
$$

Since the series in the right-hand side of (25) converges uniformly in $v$, it may be integrated term by term over $v \in[-\tau / 2, \tau / 2]$, and we obtain

$$
C_{1} \tau \omega(t, \tau) \geqslant \sum_{j=1}^{\infty} \sum_{k=1}^{\infty} k^{-1}(2 \sin j t / 2)^{m}(2 \sin k \tau / 2)^{n+1} a_{j k} \geqslant
$$




$$
\geqslant C_{2} \sum_{j=1}^{M} \sum_{k=1}^{N}(j t)^{m}(k \tau)^{n+1} k^{-1} a_{j k}
$$

and

$$
\sum_{j=1}^{M} \sum_{k=1}^{N} j^{m} k^{n} a_{j k} \leqslant C_{3} M^{m} N^{n} \omega(t, \tau) \leqslant C_{3} M^{m} N^{n} \omega(2 \pi / M, 2 \pi / N) .
$$

Step II. Let $m, n$ be even. Then, by Lemma 5 , we have

$$
C_{1} \omega(t, \tau) \geqslant\left|\dot{\Delta}_{t, \tau}^{m, n} h(0, v)\right|=\left|\sum_{j=1}^{\infty} \sum_{k=1}^{\infty} a_{j k}(2 \sin j t / 2)^{m}(2 \sin k \tau / 2)^{n} \sin k v\right| .
$$

The series in the right-hand side may be integrated term by term over $v \in[0, \tau]$, and we obtain

$$
\begin{gathered}
C_{1} \tau \omega(t, \tau) \geqslant \sum_{j=1}^{\infty} \sum_{k=1}^{\infty} k^{-1}(2 \sin j t / 2)^{m}(2 \sin k \tau / 2)^{n} a_{j k}(1-\cos k \tau)= \\
=2^{-1} \sum_{j=1}^{\infty} \sum_{k=1}^{\infty} k^{-1}(2 \sin j t / 2)^{m}(2 \sin k \tau / 2)^{n+2} a_{j k} \geqslant \\
\geqslant C_{4} \sum_{j=1}^{M} \sum_{k=1}^{N}(j t)^{m}(k \tau)^{n+2} k^{-1} a_{j k}
\end{gathered}
$$

and

$$
\sum_{j=1}^{M} \sum_{k=1}^{N} j^{m} k^{n+1} a_{j k} \leqslant C_{5} M^{m} N^{n+1} \omega(2 \pi / M, 2 \pi / N), \quad M, N \in \mathbb{N} .
$$

By the condition $\omega \in B B \cap \Delta_{2}$ (see Lemma 1) and Lemma 2 (ii), we obtain (14), while the condition $\omega \in B_{m} B_{n}$, (14) and Lemma 2 (i) imply (20).

Step III. Let $m, n$ be odd. Then, by Lemma 5 , we have

$$
\begin{gathered}
C_{1} \omega(t, \tau) \geqslant\left|\dot{\Delta}_{t, \tau}^{m, n} h(u, v)\right|= \\
=\left|\sum_{j=1}^{\infty} \sum_{k=1}^{\infty} a_{j k}(2 \sin j t / 2)^{m}(2 \sin k \tau / 2)^{n} \sin j u \cos k v\right|
\end{gathered}
$$


for $u, v \in \mathbb{R}$. The series in the right-hand side of (26) uniformly converges in $v$ and may be integrated term by term over $v \in[-\tau / 2, \tau / 2]$. Therefore,

$$
C_{1} \tau \omega(t, \tau) \geqslant\left|\sum_{j=1}^{\infty} \sum_{k=1}^{\infty} k^{-1} a_{j k}(2 \sin j t / 2)^{m}(2 \sin k \tau / 2)^{n+1} \sin j u\right|, \quad u \in \mathbb{R} .
$$

Substituting $u=t / 2$ into (27), we obtain

$$
\begin{aligned}
C_{1} \tau \omega(t, \tau) & \geqslant 2^{-1} \sum_{j=1}^{\infty} \sum_{k=1}^{\infty} k^{-1} a_{j k}(2 \sin j t / 2)^{m+1}(2 \sin k \tau / 2)^{n+1} \geqslant \\
& \geqslant C_{6} \sum_{j=1}^{M} \sum_{k=1}^{N}(j t)^{m+1}(k \tau)^{n+1} k^{-1} a_{j k}
\end{aligned}
$$

and

$$
\sum_{j=1}^{M} \sum_{k=1}^{N} j^{m+1} k^{n} a_{j k} \leqslant C_{7} M^{m+1} N^{n} \omega(2 \pi / M, 2 \pi / N), \quad M, N \in \mathbb{N} .
$$

By Lemmas 2 and 1 and the condition $\omega \in B B \cap B_{m} B_{n}$, we deduce, similarly to Step II, that (20) holds.

Step IV. Let $m$ be odd, $n$ be even. By Lemma 5, we have

$$
C_{1} \omega(t, \tau) \geqslant\left|\sum_{j=1}^{\infty} \sum_{k=1}^{\infty} a_{j k}(2 \sin j t / 2)^{m}(2 \sin k \tau / 2)^{n} \sin j u \sin k v\right|, u, v \in \mathbb{R} .
$$

Integrating (28) over $v \in[0, \tau]$, we obtain

$$
C_{1} \tau \omega(t, \tau) \geqslant\left|\sum_{j=1}^{\infty} \sum_{k=1}^{\infty} k^{-1} a_{j k}(2 \sin j t / 2)^{m}(2 \sin k \tau / 2)^{n} \sin j u(1-\cos k \tau)\right|,
$$

where $u \in \mathbb{R}$. Substituting $u=t / 2$ into (29), we obtain

$$
\begin{aligned}
C_{1} \tau \omega(t, \tau) & \geqslant 4^{-1} \sum_{j=1}^{\infty} \sum_{k=1}^{\infty} k^{-1} a_{j k}(2 \sin j t / 2)^{m+1}(2 \sin k \tau / 2)^{n+2} \geqslant \\
& \geqslant C_{8} \sum_{j=1}^{M} \sum_{k=1}^{N}(j t)^{m+1}(k \tau)^{n+2} k^{-1} a_{j k}
\end{aligned}
$$


and

$$
\sum_{j=1}^{M} \sum_{k=1}^{N} j^{m+1} k^{n+1} a_{j k} \leqslant C_{7} M^{m+1} N^{n+1} \omega(2 \pi / M, 2 \pi / N), \quad M, N \in \mathbb{N} .
$$

Using Lemmas 2 and 1 and the condition $\omega \in B B \cap B_{m} B_{n}$, we deduce, again, that (20) holds.

Step V. Secondly, we consider the general case $r, s \in \mathbb{Z}_{+}$. If $h \in W^{r, s} H^{m, n}(\omega)$, then, by Lemma 6 ,

$$
\omega_{m+r, n+s}\left(h, \delta_{1}, \delta_{2}\right) \leqslant \delta_{1}^{r} \delta_{2}^{s} \omega_{m, n}\left(h^{(r, s)}, \delta_{1}, \delta_{2}\right) \leqslant C_{5} \delta_{1}^{r} \delta_{2}^{s} \omega\left(\delta_{1}, \delta_{2}\right),
$$

i. e., $W^{r, s} H^{m, n}(\omega) \subset H^{m+r, n+s}\left(\Omega_{r, s}\right)$, where $\Omega_{r, s}\left(\delta_{1}, \delta_{2}\right)=\delta_{1}^{r} \delta_{2}^{s} \omega\left(\delta_{1}, \delta_{2}\right)$. If $\omega \in B B \cap \Delta_{2}$, then $\Omega_{r, s}$ also belongs to $B B \cap \Delta_{2}$, while if $\omega \in B_{m} B_{n}$, then, by definition, $\Omega_{r, s}$ belongs to $B_{m+r, n+s}$. Applying the results obtained in Steps I-IV in all cases, we have

$$
\begin{aligned}
& \sum_{j=1}^{M} \sum_{k=1}^{N} j^{m+r} k^{n+s} a_{j k}=O\left(M^{m+r} N^{n+s} \Omega_{r, s}(\right.\left.\left.\frac{2 \pi}{M}, \frac{2 \pi}{N}\right)\right)= \\
&=O\left(M^{m} N^{n} \omega\left(\frac{2 \pi}{M}, \frac{2 \pi}{N}\right)\right) .
\end{aligned}
$$

Thus, (20) holds under conditions of part (ii) of Theorem 1.

Corollary 1. If $m=n=1, \omega \in B B \cap B_{1} B_{1},\left\{a_{j k}\right\}_{j, k=1}^{\infty} \subset \mathbb{R}_{+}$satisfies (11) and $h(x, y)$ is the sum of (12), then the conditions

$$
\sum_{j=1}^{M} \sum_{k=1}^{N} j k a_{j k}=O(M N \omega(2 \pi / M, 2 \pi / N)), \quad M, N \in \mathbb{N},
$$

and $h \in H^{1,1}(\omega)$ are equivalent.

Corollary 2. If $m=2, n=1, \omega \in B B \cap \Delta_{2},\left\{a_{j k}\right\}_{j, k=1}^{\infty} \subset \mathbb{R}_{+}$satisfies (11) and $h(x, y)$ is the sum of (12), then the conditions

$$
\sum_{j=1}^{M} \sum_{k=1}^{N} j^{2} k a_{j k}=O\left(M^{2} N \omega(2 \pi / M, 2 \pi / N)\right), \quad M, N \in \mathbb{N},
$$

and $h \in H^{2,1}(\omega)$ are equivalent. In particular, $\omega_{2,1}\left(f, \delta_{1}, \delta_{2}\right)=O\left(\delta_{1}^{2} \delta_{2}\right)$, $\delta_{1}, \delta_{2} \in[0,2 \pi]$, if and only if the series $\sum_{j=1}^{\infty} \sum_{k=1}^{\infty} j^{2} k a_{j k}$ converges. 
Corollary 3. Let $\left\{a_{j k}\right\}_{j, k=1}^{\infty}$ and $h$ be as in Corollary 2. Then, $h \in \Lambda_{*}(2)$ (see Introduction) if and only if the condition

$$
\sum_{j=M}^{\infty} \sum_{k=N}^{\infty} a_{j k}=O\left(M^{-1} N^{-1}\right), \quad M, N \in \mathbb{N},
$$

holds.

Remark. Corollary 3 is obtained by Fülöp [9] together with its "small" analogue that may be derived from Theorem 6. Let us note that a particular case $\omega\left(\delta_{1}, \delta_{2}\right)=\delta_{1}^{2} \delta_{2}$ in Corollary 2 corresponds to the exclusive case (ii) in Theorem 1.

5. Concluding remarks. We say that $\left\{a_{j k}\right\}_{j, k=1}^{\infty}$ is weak monotone if $a_{j k} \geqslant 0$ for all $j, k$ and $a_{i j} \leqslant C a_{k l}$ for all $i \in[k, 2 k-1], j \in$ $[l, 2 l-1]$. The famous Lorentz theorem [13] states that if $\left\{a_{n}\right\}_{n=1}^{\infty}$ decreases to zero and $0<\alpha<1$, then the assertions (i) $a_{n}=O\left(n^{-\alpha-1}\right), n \in \mathbb{N}$, (ii) $f(x)=\sum_{n=1}^{\infty} a_{n} \cos n x \in \operatorname{Lip}(\alpha)$ and (iii) $g(x)=\sum_{n=1}^{\infty} a_{n} \sin n x \in \operatorname{Lip}(\alpha)$ are equivalent. Several one-dimensional generalizations of the Lorentz theorem to generalized Lipschitz or Nikol'skii spaces and classes of general monotone sequences may be found in papers of Tikhonov [23] and [24]. The following theorem is an extension of the Lorentz result and results from [23] to the two-dimensional mixed case. Applications of onedimensional weak monotonicty can be found in [12]. Also, we note the paper by Dyachenko and Tikhonov [7], where the estimates of Fourier coefficients satisfying another definition of weak monotonicity are given.

Theorem 5. Let $\left\{a_{j k}\right\}_{j, k=1}^{\infty}$ be weak monotone, $m, n \in \mathbb{N}, r, s \in \mathbb{Z}_{+}$, $\omega \in B B \cap B_{m} B_{n}$. If $\left\{a_{j k}\right\}_{j, k=1}^{\infty}$ satisfies $(11), h(x, y)$ is the sum of (12), then the conditions

(i) $h \in W^{r, s} H^{m, n}(\omega)$;

and

(ii) $a_{j k}=O\left(j^{-r-1} k^{-s-1} \omega(2 \pi / j, 2 \pi / k)\right), j, k \in \mathbb{N}$, are equivalent.

The proof of Theorem 5 is similar to the proof of Theorem 2 from [27].

The last theorem is the $o$-analog of Theorem 4 .

Theorem 6. (i) Let $m, n \in \mathbb{N}, r, s \in \mathbb{Z}_{+}, \omega \in B B \cap \Delta_{2}$. If $\left\{a_{j k}\right\}_{j, k=1}^{\infty}$ satisfies (11), $h$ is the sum of (12) and the conditions (20) and

$$
\sum_{j=1}^{M} \sum_{k=1}^{N} j^{m+r} k^{n+s}\left|a_{j k}\right|=o\left(M^{m} N^{n} \omega(2 \pi / M, 2 \pi / N)\right), \quad M, N \rightarrow \infty,
$$


are valid, then $h \in W^{r, s} h^{m, n}(\omega)$.

(ii) If $m, n \in \mathbb{N}, r, s \in \mathbb{Z}_{+}, a_{j, k} \geqslant 0$ for all $j, k \in \mathbb{N},\left\{a_{j k}\right\}_{j, k=1}^{\infty}$ satisfies (11), $h$ is the sum of (12), $\omega \in B B \cap \Delta_{2}, m+r$ is even, $n+s$ is odd, and $h \in W^{r, s} h^{m, n}(\omega)$, then (30) is valid. If, in addition to the previous conditions of (ii), we have $\omega \in B_{m} B_{n}$, then also (30) is valid.

Proof. Similarly to the proof of Theorem 4, we use the estimate (21) for $t, \tau \in(0,2 \pi]$ and $M=[2 \pi / t], N=[2 \pi / \tau]$. By (30) and the condition $\omega \in \Delta_{2}$, we find that

$$
\begin{gathered}
I_{M N}^{(1)} \leqslant \sum_{j=1}^{M} \sum_{k=1}^{N} j^{r} k^{s}\left|a_{j k}\right|(j t)^{m}(k \tau)^{n} \leqslant \\
\leqslant \varepsilon t^{m} \tau^{n} M^{m} N^{n} \omega(2 \pi / M, 2 \pi / N) \leqslant C_{1} \varepsilon \omega(t, \tau), \quad 0<t, \tau<\delta_{1}(\varepsilon) .
\end{gathered}
$$

By Lemma 4 (ii) applied to $\left\{j^{r} k^{s} a_{j k}\right\}_{j, k=1}^{\infty}$ and (30) for $M, N>n_{0}(\varepsilon$ ), we obtain

$$
I_{M N}^{(2)} \leqslant C_{2} \tau^{n} \sum_{j=M+1}^{\infty} \sum_{k=1}^{N} j^{r} k^{n+s} a_{j k}<\varepsilon \tau^{n} N^{n} \omega\left(\frac{2 \pi}{M}, \frac{2 \pi}{N}\right) \leqslant C_{3} \varepsilon \omega(t, \tau),
$$

where $0<t, \tau<\delta_{2}(\varepsilon)$. The quantity $I_{M N}^{(3)}$ is estimated in a similar manner for $0<t, \tau<\delta_{3}(\varepsilon)$. By the condition $\omega \in B B \cap \Delta_{2}$ and Lemma 3 (ii), we have $I_{M N}^{(4)}=o\left(M^{m} N^{n} \omega(2 \pi / M, 2 \pi / N)\right), M, N \rightarrow \infty$, whence

$$
I_{M N}^{(4)} \leqslant C_{4} \varepsilon \omega(t, \tau), \quad 0<t, \tau<\delta_{4}(\varepsilon) .
$$

From estimates (31)-(33) we find that $\left|\dot{\Delta}_{t, \tau}^{m, n} h^{(r, s)}(x, y)\right| \leqslant C_{5} \varepsilon \omega(t, \tau)$ for all $t, \tau<\delta(\varepsilon):=\min _{1 \leqslant i \leqslant 4} \delta_{i}(\varepsilon)$, i. e. $h \in W^{r, s} h^{m, n}(\omega)$.

(ii) The assertion is proved similarly to the proof of (ii) from Theorem 4. We may substitute in all steps of this proof $C_{1}$ instead of $\eta$ and get at the end of Steps I-IV an estimate of type $K \eta M^{m} N^{n} \omega(2 \pi / M, 2 \pi / N)$, where $K$ depends on $m, n$. Setting $K \eta=\varepsilon$, we finish the proof.

Remark. It is interesting to obtain a variant of Theorem 4 without the condition $\omega \in B_{m} B_{n}$. The first attempt in this direction may be found in [27, Theorem 3], but this result gives only sufficient conditions for an odd in each argument functions to belong to a Nikol'skii class.

Acknowledgement. Author thanks all referees for their valuable comments improving the paper. 


\section{References}

[1] Bary N. K., Stechkin S. B. Best approximations and differential properties of two conjugate functions. Trudy Moskov. Mat. Obshch., 1956, vol. 5, pp. 483-522 (in Russian).

[2] Boas R. P. Fourier series with positive coefficients. J. Math. Anal. Appl. 1967, vol. 17, no. 3, pp. 463-483.

DOI: https://doi.org/10.1016/0022-247X (67)90134-5

[3] Butzer P. L., Dyckhoff H., Görlich E., Stens R. L. Best trigonometric approximations, fractional order derivatives and Lipschitz classes. Canad. J. Math., 1977, vol. 29, no. 4, pp. 781-793.

DOI: https://doi.org/10.4153/CJM-1977-081-6

[4] Chan L. Y. On Fourier series with non-negative coefficients and two problems of R. P. Boas. J. Math. Anal. Appl., 1985, vol. 110, no. 1, pp. 116-129. DOI: https://doi.org/10.1016/0022-247X (85) 90338-5

[5] Donskikh S. L. Multiple Fourier series for functions from a Zygmund class. Moscow Univ. Math. Bull., 2010, vol. 65, no. 1, pp. 1-9.

DOI: https://doi.org/10.3103/S0027132210010018.

[6] Dyachenko M. I. Trigonometric series with generalized monotone coeffcients. Izv. Vyssh. Uchebn. Zaved. Mat., 1986, no. 7, pp. 39-50.

[7] Dyachenko M., Tikhonov S. Smoothness and asymptotic properties of functions with general monotone Fourier coefficients. J. Fourier Anal. Appl., 2018, vol. 24, no. 4, pp. 1072-1097.

DOI: https://doi.org/10.1007/s00041-017-9553-7

[8] Fülöp V. Double cosine series with nonnegative coefficients. Acta Sci. Math.(Szeged), 2004, vol. 70, no. 1-2, pp. 91-100.

[9] Fülöp V. Double sine and cosine-sine series with nonnegative coefficients. Acta Sci. Math.(Szeged), 2004, vol. 70, no. 1-2, pp. 101-116.

[10] Fülöp V., Móricz F. Absolutely convergent multiple Fourier series and multiplicative Zygmund classes of functions. Analysis, 2008, vol. 28, no. 3, pp. 345-354. DOI: https://doi.org/10.1524/anly.2008.0919

[11] Han D., Li G., Yu D. Double sine series and higher order Lipschitz classes of functions. Bull. Math. Anal. Appl., 2013, vol. 5, no. 1, pp. 10-21.

[12] Liflyand E., Tikhonov S., Zeltser M. Extending tests for convergence of number series. J. Math. Anal. Appl., 2011, vol. 377, no. 1, pp. 194-206.

DOI: https://doi.org/10.1016/j.jmaa.2010.10.007

[13] Lorentz G. G. Fourier-Koeffizienten und Funktionenklassen. Math. Zeitschr., 1948, vol. 51, no. 2, pp. 135-149.

DOI: https://doi.org/10.1007/BF01290998 
[14] Móricz F. Absolutely convergent multiple Fourier series and multiplicative Lipschitz classes of functions. Acta Math. Hung., 2008, vol. 121, no. 1-2, pp. 1-19. DOI: https://doi.org/10.1007/s10474-008-7164-0

[15] Németh J. Note on the Fourier series with nonnegative coefficients. Acta Sci. Math.(Szeged), 1991, vol. 55, no. 1-2, pp. 83-93.

[16] Németh J., On the Fourier series with nonnegative coefficients. Acta Sci. Math.(Szeged), 1991, vol. 55, no. 1-2, pp. 95-101.

[17] Potapov M. K., Simonov B. V., Tikhonov S. Yu. On the Besov and BesovNikolskii classes and on estimates for the mixed moduli of smoothness of fractional derivatives. Proc. Steklov Inst. Math., 2003, vol. 243, pp. 234-246; translation from Tr. Mat. Inst. Steklova, 2003, vol. 243, pp. 244-256.

[18] Potapov M. K., Simonov B. V., Tikhonov S. Yu. Mixed moduli of smoothness in $L_{p}, 1<p<\infty$ : a survey. Surveys in Appr. Theory, 2013, vol. 8, pp. $1-57$.

[19] Rubinstein A. I. On $\omega$-lacunary series and functions from classes $H^{\omega}$. Mat. sb., 1964, vol. 65(107), no. 2, pp. 239-271 (in Russian).

[20] Tevzadze T. Sh. On certain classes of functions and Fourier series. Trudy Tbilisskogo universiteta (Proceedings of Tbilisi Univ.), 1973, vol. 149-150, pp. 51-64 (in Russian).

[21] Tikhonov S. Smoothness conditions and Fourier series. Math. Ineq. Appl., 2007, vol. 10, no. 2, pp. $229-242$.

DOI: https://doi.org/10.7153/mia-10-19

[22] Tikhonov S. On generalized Lipschitz classes and Fourier series. Zeichr. Anal. Anwend., 2004, vol. 23, no. 4, pp. 745-764.

DOI: https://doi.org/10.4171/ZAA/1220

[23] Tikhonov S. Trigonometric series of Nikol'skii classes. Acta Math. Hungar., 2007, vol. 114, no. 1-2, pp. 61-78.

DOI: https://doi.org/10.1007/s10474-006-0513-y

[24] Tikhonov S. Best approximation and moduli of smoothness: computation and equivalence theorems. J. Approx. Theory, 2008, vol. 153, no. 1, $19-39$. DOI: https://doi.org/10.1016/j.jat.2007.05.006

[25] Timan A. F. Theory of approximation of functions of a real variable. Macmillan, New York, 1963.

[26] Volosivets S. S. Multiple Fourier coefficients and generalized Lipschits classes in uniform metric. J. Math. Anal. Appl., 2015, vol. 427, no. 2, pp. 1070 -1083. DOI: https://doi.org/10.1016/j.jmaa.2015.02.011

[27] Volosivets S. S. Multiple sine series and Nikol'skii classes in uniform metric. European J. Math., 2019, vol. 5, no. 1, pp. 206-222.

DOI: https://doi.org/10.1007/s40879-018-0226-0 
[28] Yu D. Double trigonometric series with positive coefficients. Anal. Math., 2009, vol. 35, no. 2, pp. $149-167$.

DOI: https://doi.org/10.1007/s10476-009-0205-2

[29] Zygmund A. Trigonometric series. vol. 2. Cambridge Univ. Press, Cambridge, 1959.

Received July 03, 2019.

In revised form, September 20, 2019.

Accepted September 24, 2019.

Published online October 7, 2019.

Saratov State University

83 Astrakhanskaya St.,

Saratov 410012, Russia

E-mail: VolosivetsSS@mail.ru 